\title{
Motion-Based Design of Semi-active Tuned Mass Dampers to Control Pedestrian-Induced Vibrations in Footbridges Under Uncertainty Conditions
}

\author{
Javier Fernando Jiménez-Alonso $^{1(\bowtie)}$ (D), José Manuel Soria Herrera ${ }^{1}$ (D), \\ Carlos Martín de la Concha Renedo ${ }^{1}$ (D), and Francisco Guillen-González ${ }^{2}$ \\ 1 Escuela Técnica Superior de Ingenieros de Caminos, Canales y Puertos, \\ Universidad Politécnica de Madrid, 28040 Madrid, Spain \\ jf.jimenez@upm.es \\ 2 Facultad de Matemáticas, Universidad de Sevilla, 41012 Sevilla, Spain
}

\begin{abstract}
Modern slender footbridges are sensitive to human-induced vibrations together with the uncertainty associated with the variation of the operational and environmental conditions. In order to overcome these limitations, semi-active damping devices have been widely employed due to their adequate balance between their effectiveness and their cost when they are used to control the pedestrian-induced vibrations in footbridges. Different design methods have been proposed to guarantee that the footbridges, controlled by these damping devices, meet the vibration serviceability limit state without compromising their budget. Among these proposals, the motion-based design method has shown a high performance when it has been implemented to design passive damping devices for footbridges. Herein, the motion-based design method under uncertainty conditions has been adapted and further implemented for the robust optimum design of semi-active tuned mass dampers when they are employed to control the pedestrianinduced vibrations in slender footbridges. According to this method, the design problem can be transformed into two sub-problems: (i) a multi-objective optimization sub-problem; and (ii) a reliability analysis sub-problem. Thus, its main objective is to find the parameters of the semi-active damping device which guarantee an adequate comfort level without compromising its cost. In order to take into account the effect of the modification of the structural modal properties associated with the variation of the operational and environmental conditions, the compliance of the design requirements has been formulated via a reliability index. Therefore, a reliability analysis must be performed to assess the probability of failure associated with the abovementioned serviceability limit state.
\end{abstract}

Keywords: Motion-based design $\cdot$ Structural control $\cdot$ Semi-active tuned mass damper · Pedestrian-induced vibrations · Footbridges · Uncertainty conditions 


\section{Introduction}

The improvement of the strength of the construction materials together with the current aesthetic requirements of the modern societies have increased the slenderness of footbridges [1]. Accordingly, this higher slenderness has increased the sensitivity of footbridges to two phenomena: (i) the human-structure interaction [2] and (ii) the variability of their modal properties associated with the modification of their operational and environmental conditions [3].

In order to overcome these limitations, external damping devices have been widely installed on these structures to guarantee their robust structural behavior during their overall life cycle [4]. Among these damping devices, semi-active damping systems have shown an adequate balance between their effectiveness and cost when they are implemented under stochastic conditions [5]. Different algorithms and control laws have been proposed to address successfully the design process of these damping devices [6]. Among these proposals, the motion-based design method under a stochastic approach is presented and further implemented herein to design semi-active tuned mass dampers (STMD) when they are employed to control the pedestrian-induced vibrations in slender footbridges under uncertainty conditions [7]. Thus, the main contribution of this study is to adapt the abovementioned method, which has been previously implemented for the design of different passive damping systems [8], for the design of STMDs.

According to this method, the design problem may be transformed into two coupled sub-problems: (i) a multiple-objective optimization sub-problem [9]; and (ii) a reliability analysis sub-problem [10]. The objective function of the first sub-problem is defined in terms of two different elements: (i) the parameters of the STMD which are needed to be determined; and (ii) the design requirements of the structure which are needed to be met [2]. As the main objective of this design process is to control the pedestrian-induced vibrations in footbridges, the design requirements may be defined in terms of the comfort level of the footbridge. Thus, these design requirements are met if the maximum accelerations of the structure are lower than an allowable acceleration established by the designer [2]. Additionally, due to the sensitivity of the modal properties of the structure to the variation of the operational and environmental conditions, the abovementioned design requirements have been re-formulated following a probabilistic approach. According to this approach, the design requirements are met if a reliability index, $\beta$, which reflects the probability of compliance of the abovementioned vibration serviceability limit state (VSLS), is greater than an allowable reliability index, $\beta_{\text {lim }}$ [10]. Sampling techniques are usually considered to compute this reliability index [10]. Herein a conventional Monte Carlo method has been regarded [10]. Finally, the performance of the proposed algorithm has been validated via the analysis of a numerical case-study [2]. Concretely, the VSLS of a steel footbridge under uncertainty conditions has been met via the installation of a STMD designed according to the proposed algorithm.

The paper is organized as follows. In Sect. 2, the mathematical model of the STMDfootbridge interaction system is formulated and its dynamic response under pedestrian action is computed numerically in time domain. Subsequently, in Sect. 3, the motionbased design method under a stochastic approach is described in detail. Later, in Sect. 4, a numerical case-study is presented to assess the performance of the proposed method when it is implemented for the design of a STMD employed to control the dynamic 
response of a steel footbridge under pedestrian action. Finally, some concluding remarks are included in Sect. 5 to finish the paper.

\section{Semi-active Tuned Mass Damper-Footbridge Interaction Model Under Pedestrian Load}

In order to design the semi-active damping device according to the mentioned method, the dynamic response of a STMD-footbridge interaction system under pedestrian action must be assessed. For this purpose, the following steps must be given: (i) the formulation of a STMD-footbridge interaction model; (ii) the definition of the pedestrian load; (iii) the definition of the driving force (a control law); and (iv) the numerical integration of the equations of motion which governs the STMD-footbridge interaction model.

For the formulation of the STMD-footbridge interaction model, the following assumptions have been considered herein: (i) the behavior of the structure (the footbridge) is simulated via a single vibration mode (modal coordinates) since it is assumed that only a vibration mode is prone to suffer from pedestrian-induced vibrations [11]; (ii) the STMD is modelled via a single degree of freedom system (physical coordinates); (iii) the pedestrian load is simulated by an equivalent harmonic load; and (iv) the STMD is located at the point with the maximum modal displacement. Figure 1 shows a scheme of the STMD-footbridge interaction model.

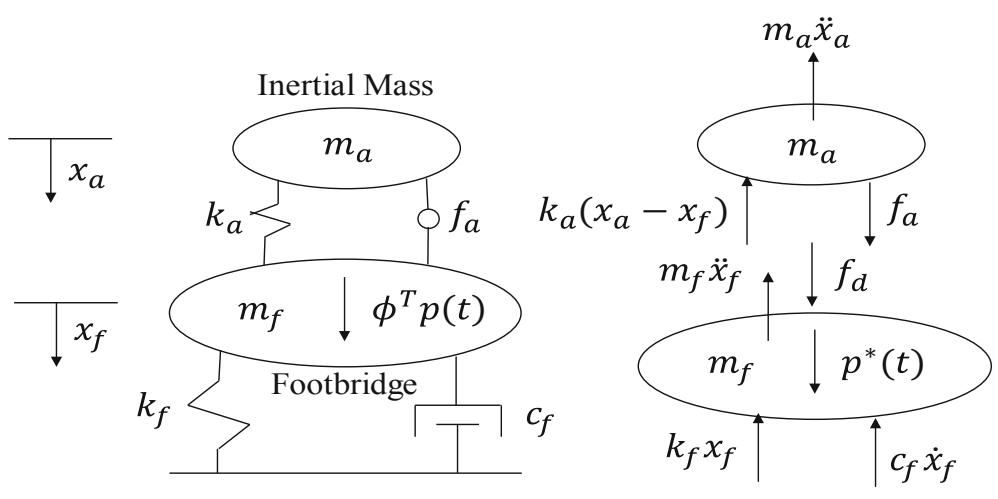

Fig. 1. STMD-footbridge interaction model.

Thus, the equations of motion of the STMD-footbridge interaction model can be obtained via the implementation of the second Newton's law to the two masses (STMD and equivalent modal mass). These equations may be expressed as follows:

$$
\begin{gathered}
m_{f} \ddot{x}_{f}(t)+c_{f} \dot{x}_{f}(t)+k_{f} x_{f}(t)=p^{*}(t)+f_{d}(t) \\
m_{a} \ddot{x}_{a}(t)+k_{a}\left(x_{a}(t)-x_{f}(t)\right)=f_{a}(t)
\end{gathered}
$$




$$
f_{d}(t)=k_{a}\left(x_{a}(t)-x_{f}(t)\right)-f_{a}(t)=-m_{a} \ddot{x}_{a}(t)
$$

where $m_{f}[\mathrm{~kg}], c_{f}[\mathrm{sN} / \mathrm{m}]$ and $k_{f}[\mathrm{~N} / \mathrm{m}]$ are respectively the mass, damping and stiffness of the considered vibration mode of the footbridge; $p^{*}(t)=\phi^{T} p(t)[\mathrm{N}]$ is the projection of the pedestrian load on the considered vibration (being $p(t)$ the pedestrian load $[\mathrm{N}], \phi$ the considered vibration mode and $T$ the transpose function); $\ddot{x}_{f}(t)\left[\mathrm{m} / \mathrm{s}^{2}\right]$, $\dot{x}_{f}(t)[\mathrm{m} / \mathrm{s}]$ and $x_{f}(t)[\mathrm{m}]$ are respectively the acceleration, velocity and displacement of the footbridge; $m_{a}[\mathrm{~kg}]$ and $k_{a}[\mathrm{~N} / \mathrm{m}]$ are respectively the mass and stiffness of the STMD, $\ddot{x}_{a}(t)\left[\mathrm{m} / \mathrm{s}^{2}\right]$ and $x_{a}(t)[\mathrm{m}]$ are respectively the acceleration and displacement of the STMD; $f_{a}(t)[\mathrm{N}]$ is the driving force generated by the semi-active damper and $f_{d}(t)$ $[\mathrm{N}]$ is the control force generated by the STMD.

These equations can be re-organized in matrix form as follows:

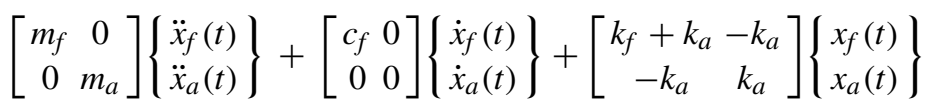

$$
\begin{aligned}
& =\left\{\begin{array}{l}
1 \\
0
\end{array}\right\} p^{*}(t)+\left\{\begin{array}{c}
-1 \\
1
\end{array}\right\} f_{a}(t) \\
& {[M]\{\ddot{x}(t)\}+[C]\{\dot{x}(t)\}+[K]\{x(t)\}=\left\{B_{0}\right\} p^{*}(t)+\left\{B_{c}\right\} f_{a}(t)}
\end{aligned}
$$

where $[M]=\left[\begin{array}{cc}m_{f} & 0 \\ 0 & m_{a}\end{array}\right]$ is the mass matrix; $[C]=\left[\begin{array}{cc}c_{f} & 0 \\ 0 & 0\end{array}\right]$ is the damping matrix; $[\mathrm{K}]=\left[\begin{array}{cc}k_{f}+k_{a}-k_{a} \\ -k_{a} & k_{a}\end{array}\right]$ is the stiffness matrix; $\left\{B_{0}\right\}$ is the input vector associated with the pedestrian load; $\left\{B_{c}\right\}$ is the input vector associated with the driving force; $\{\ddot{x}(t)\}$ is the acceleration vector; $\{\dot{x}(t)\}$ is the velocity vector and $\{x(t)\}$ is the displacement vector.

In order to integrate this equation system and make easier the implementation of a control law for the determination of the driving force, $f_{a}(\mathrm{t})$, the above-mentioned equation system has been transformed into a state space formulation [11]. According to this formulation, the dynamic behavior of a linear invariant system may be expressed by the following differential equation system with initial conditions $\left(\{z(0)\}=z_{0}\right)$ :

$$
\begin{gathered}
\{\dot{z}(t)\}=[A]\{z(t)\}+[B]\left\{p^{*}(t)\right\}+[B]\left\{f_{a}(t)\right\} \\
\{y(t)\}=[E]\{z(t)\}+[D]\left\{f_{a}(t)\right\}
\end{gathered}
$$

where $\{z(t)\}$ is the state vector; $\{y(t)\}$ is the output vector; $[A]$ is the system matrix; $[B]$ is the input matrix; $[E]$ is the output matrix; and $[D]$ is the feedthrough matrix [11]. It is possible via matrix transformations to convert the abovementioned equation system into its state space formulation [11]. The state space matrices can be defined as:

$$
\begin{gathered}
{[A]=\left[\begin{array}{cc}
0 & I \\
-[M]^{-1}[K]-[M]^{-1}[C]
\end{array}\right]} \\
{[B]=\left[\begin{array}{c}
0 \\
-[M]^{-1}\left\{B_{i}\right\}
\end{array}\right] \text { where }\left\{B_{i}\right\}=\left\{\begin{array}{l}
\left\{B_{0}\right\} \text { for } p^{*}(t) \\
\left\{B_{c}\right\} \text { for } f_{a}(t)
\end{array}\right.}
\end{gathered}
$$




$$
\begin{gathered}
{[E]=-\left[E_{a}\right]\left[[M]^{-1}[K][M]^{-1}[C]\right]} \\
{[D]=\left[E_{a}\right][M]^{-1}[B]}
\end{gathered}
$$

where the state vector $\{z(t)\}=\left\{x_{f}(t) x_{a}(t) \dot{x}_{f}(t) \dot{x}_{a}(t)\right\}$ is defined in terms of the displacements and velocities of both the footbridge and the STMD; and the output vector, $\{y(t)\}$, is defined in terms of the accelerations experienced by the footbridge (being $\left[E_{a}\right]$ the acceleration matrix which indicates the elements in which the acceleration is computed).

In order to obtain the response of the above state space equation system both the pedestrian force, $p(t)$, and the driving force, $f_{a}(t)$, which simulates the behavior of the semi-active damper, must be defined.

The pedestrian force, $p(t)$, has been simulated according to the recommendations of the French guidelines [2]. Only the vertical contribution of the walking pedestrian action has been considered herein. According to these guidelines, the walking pedestrian action, $p(t)$, can be determined via an equivalent harmonic force defined as follows:

$$
p(t)=280 \cdot \cos \left(2 \pi \cdot f_{f} \cdot t\right) \cdot n_{e q} \cdot \psi
$$

where $f_{f}[\mathrm{~Hz}]$ is the natural frequency of the considered vibration mode of the footbridge; $n_{e q}[-]$ is the equivalent number of pedestrians; and $\psi$ is a reduction factor which takes into account the probability that the natural frequency is within the range which characterizes the pedestrian-structure interaction in vertical direction $\left(1.25 \leq f_{f} \leq 2.3 \mathrm{~Hz}\right)$ [2].

The driving force, $f_{a}(t)$, is determined via the implementation of a feedback controller to the above mentioned system in the state space [11]. Figure 2 shows the general layout of the feedback controller considered.

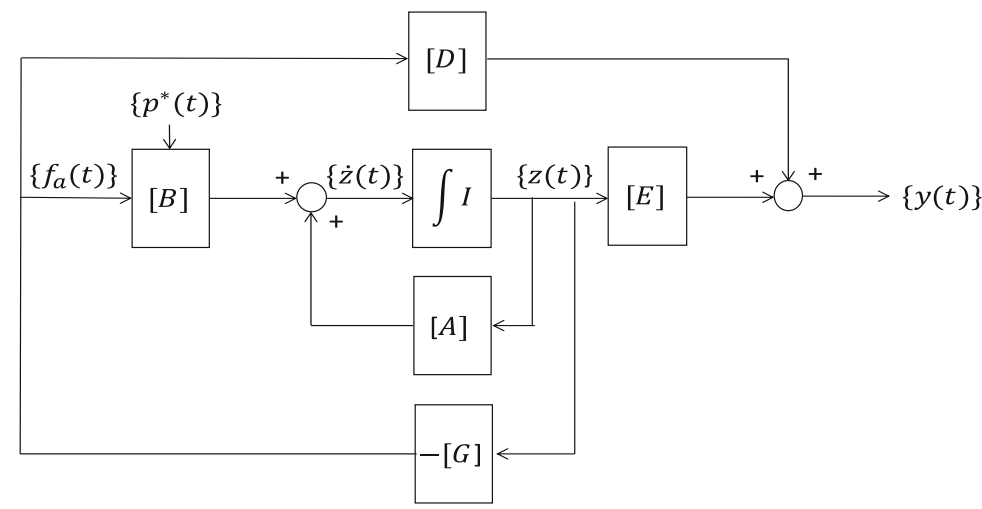

Fig. 2. Design of a feedback controller in a state space formulation [11].

The implementation of this feedback controller allows modifying the system equation as follows:

$$
\left.\{\dot{z}(t)\}=[A]\{z(t)\}+\left[B_{0}\right]\left\{p^{*}(t)\right\}+\left[B_{c}\right]\left\{f_{a}(t)\right\}\right]
$$


where $\left[B_{0}\right]$ and $\left[B_{c}\right]$ are obtained from Eq. (9) considering as pattern load vectors $\left\{B_{0}\right\}$ and $\left\{B_{c}\right\}$ respectively.

Thus, according to the feedback controller (Fig. 2), the driving force, $f_{a}(t)$, may be determined in terms of a gain matrix, $-[G]$, and the state vector, $\{z(t)\}$. Thus, the system equation may be expressed as:

$$
\begin{gathered}
\{\dot{z}(t)\}=[A]\{z(t)\}+\left[B_{0}\right]\left\{p^{*}(t)\right\}-\left[B_{c}\right][G]\{z(t)\} \\
\{\dot{z}(t)\}=\left([A]-\left[B_{c}\right][G]\right)\{z(t)\}+\left[B_{0}\right]\left\{p^{*}(t)\right\}
\end{gathered}
$$

There are several algorithms [11] to determine the value of the gain matrix, $-[G]$. Among these algorithms, the linear quadratic regular (LQR) method [11] has been considered herein due to its extensive use for practical engineering applications. According to this method, the value of the gain matrix, $-[G]$, is obtained via the minimization of the following performance-index function, $J$.

$$
J=\int_{0}^{\infty}\left[\{z(t)\}^{T}[Q]\{z(t)\}+\{[G]\{z(t)\}\}^{T}[R]\{[G]\{z(t)\}\}\right] d t
$$

where $[Q]$ and $[R]$ are two weighting matrices which may be computed in terms of the mass, $[M]$, and stiffness, $[K]$, matrices of the interaction model [11]. According to the proposals of several authors [11], the weighting matrices may be determined as follows:

$$
\begin{gathered}
{[Q]=\alpha_{d}\left[\begin{array}{cc}
{[K]} & 0 \\
0 & {[M]}
\end{array}\right]} \\
{[R]=\beta_{d}[I]}
\end{gathered}
$$

where $\alpha_{d}[-]$ and $\beta_{d}$ [-] are the weighting factors. In this manner, once the value of these factors has been fixed, the value of the different element of the gain matrix, $-[G]$, which minimizes the value of the performance-index function, $J$, is obtained.

Additionally, in each time step, the driving force, $f_{a}(t)$, determined by the LQR controller, must be modified by a clipped force algorithm [11]. This algorithm adapts the behavior of a general actuator to the particular limitations of a semi-active damper. Thus, the driving force, $f_{a}(t)$, is bounded between a minimum, $f_{\min }[\mathrm{N}]$, and a maximum, $f_{\max }$ $[\mathrm{N}]$, force which reflect the extreme values of the constitutive model of a semi-active damper (for instance a magneto-rheological damper). For this purpose, the following relationship has been considered herein:

$$
f_{a}(t)=\left\{\begin{array}{cc}
\operatorname{sgn}\left(f_{l q r}(t)\right) \cdot f_{\text {max }} & f_{l q r}(t) \cdot \dot{u}_{r}(t)<0 \&\left|f_{l q r}(t)\right|>f_{\text {max }} \\
f_{l q r} & \text { if } \quad f_{l q r}(t) \cdot \dot{u}_{r}(t)<0 \& f_{\text {min }}<\left|f_{l q r}(t)\right|<f_{\text {max }} \\
\operatorname{sgn}\left(f_{l q r}(t)\right) \cdot f_{\text {min }} & f_{l q r}(t) \cdot \dot{u}_{r}(t)>0 \&\left|f_{l q r}(t)\right|<f_{\text {min }}
\end{array}\right.
$$

where $\dot{u}_{r}(t)[\mathrm{m} / \mathrm{s}]$ is the relative velocity of the semi-active damper; $\operatorname{sgn}()$ is the sign function; and $f_{l q r}(t)[\mathrm{N}]$ is the driving force computed by the LQR controller.

Subsequently, the response of the STMD-footbridge interaction model has been assessed via the integration of the abovementioned state space system using a RungeKutta method, as it is implemented in the Matlab software [12]. 
To sum up, the adjustment of the driving force, $f_{a}(t)$, is addressed via the compliance of the following steps: (i) to develop a linear model of the STMD-footbridge interaction model in the state space domain (where the value of the mass and stiffness of the STMD are fixed); (ii) to adjust the value of the weighting matrices, $[Q]$ and $[R]$ in terms of the weighting factors, $\alpha_{d}$ and $\beta_{d}$; (iii) to determine the optimum value of the driving force, $f_{a}(t)$, via the minimization of the performance-index function, $J$, and the clipped force algorithm; (iv) to simulate numerically the response of the linear system; and (v) to modify the values of the weighting factors and repeat the steps (ii) to (iv) until the dynamic response of the footbridge meets the design requirements [2] (established by the designer).

\section{Motion-Based Design Under Uncertainty Conditions}

The abovementioned control algorithm has been hybridized with the motion-based design method under uncertain conditions [8] to improve the performance of the design process of semi-active damping devices. The performance-based design method [13] transforms the design problem into two coupled sub-problems: (i) a multi-objective optimization sub-problem; and (ii) a reliability analysis sub-problem. Thus, the main objective of this design problem is to determine the parameters of the STMD that, minimizing its cost, ensures the compliance of the design requirements [2]. As the design requirements, which need to be accomplished, are defined in terms of the accelerations of the structure, $\ddot{x}_{f}(t)$, this design process may be understood as a motion-based design optimization method [7].

Therefore, the general formulation of the proposed method may be expressed as follows [13]:

$$
\begin{gathered}
\text { Find } \theta_{i} i=1, \ldots, n_{d} \\
\text { Minimizing } \mathbf{f}\left(\theta_{i}\right)=\left[f_{1}\left(\theta_{i}\right) \ldots f_{j}\left(\theta_{i}\right) \ldots f_{n_{f}}\left(\theta_{i}\right)\right]
\end{gathered}
$$

Subjected to $\theta_{i}^{l} \leq \theta_{i} \leq \theta_{i}^{u}$

where $\mathbf{f}\left(\theta_{i}\right)$ is the multi-objective function; $f_{j}\left(\theta_{i}\right)$ is the $j^{\text {th }}$ terms of the multi-objective function; $n_{f}$ is the number of terms of multi-objective function; $\theta_{i}$ is the $i^{\text {th }}$ design variable; $\theta_{i}^{l}$ is the lower bound of the $i^{t h}$ design variable; $\theta_{i}^{u}$ is the upper bound of the $i^{t h}$ design variables, and $n_{d}$ is the total number of design variables.

For this case, the design of a STMD, the above multi-objective function may be formulated in terms of two aspects: (i) the different parameters of the STMD; and (ii) the compliance of the VSLS of the footbridge. According to the most advances guidelines [2], the VSLS is met if the maximum acceleration of the structure, $\ddot{x}_{f}(t)$, is lower than an allowable value, $\ddot{x}_{f, \text { lim }}$, established by the designer [2].

Additionally, in order to take into account the random character of the modal properties of the footbridge, which are sensitive to the variation of the operational and environmental conditions [3], the VSLS is re-formulated via a probabilistic approach [10]. According to this approach, the modal properties of the footbridge are assumed to be random variables which follow normal probability distribution function. Hence, the 
response of the system is also a random variable, and it is possible to determine the probability of failure associated with the compliance of the different design requirements (in this case, the VSLS). According to this, the VSLS is defined in terms of a reliability index, $\beta$, which establishes certain probability of failure of its compliance. Thus, this limit state is met if this reliability index, $\beta$, is greater than an allowable value, $\beta_{\text {lim }}$, established by the design guidelines [14]. In order to compute the reliability index, $\beta$, analytical and numerical method can be used [10]. Among these methods, a Monte Carlo simulation has been considered herein [10].

In order to establish the formulation of this design problem, according to the motionbased design, the design parameters must be determined. For this particular case, four are the design variables: (i) the mass ratio, $\mu=m_{a} / m_{f}[-]$; (ii) the frequency ratio, $\delta=f_{a} / f_{f}[-]$ (where $f_{a}[\mathrm{~Hz}]$ is the natural frequency of the STMD); and (iii) the weighting factors, $\alpha_{d}$ and $\beta_{d}$ (the driving force). In order to reduce the number of design variables, simplifying the problem, a hybrid strategy has been considered herein. Thus, the frequency ratio, $\delta$, of the STMD has been selected following a conventional criterion for the adjustment of tuned mass dampers [2]. According to this criterion, the frequency ratio of the STMD is set as, $\delta=1 /(1+\mu)$.

In this manner, the formulation of the motion-based design method under uncertainty conditions for this particular problem may be formulated as follows:

$$
\begin{gathered}
\text { Find } \mu, \alpha_{d}, \beta_{d} \\
\text { Minimizing }\left\{\mathbf{f}\left(\mu, \alpha_{d}, \beta_{d}\right)\right\}=\left\{f_{1}(\mu) f_{2}\left(\mu, \alpha_{d}, \beta_{d}\right)\right\}=\left\{\mu \frac{\beta_{l i m}}{\beta}\right\} \\
\text { Subjected to } \mu \in[0.010 .10] \alpha_{d} \in[101000] \beta_{d} \in\left[10^{-5} 10^{-8}\right]
\end{gathered}
$$

Subsequently, in order to solve this optimization problem, global optimization algorithms are normally employed due to their good efficiency to find optimum solutions in nonlinear optimization problems [9]. Among these computational algorithms, a natureinspired computational algorithm, genetic algorithms, has been considered herein [9]. As result of this optimization process, a set of non-dominated solutions is obtained [9]. This set of solutions may be represented in a functional space, generating the so-called Pareto front [9]. Finally, a subsequent decision making problem must be addressed, the selection of the best solution among the different elements of the Pareto front. Therefore, an additional condition, $\frac{\beta_{\lim }}{\beta}$, has been included herein; to select the best element of the Pareto front.

\section{Application Example: Motion-Based Design of a STMD for a Vibrating Footbridge Under Uncertainty Conditions}

In order to illustrate the performance of the motion-based design method, when it is implemented to design STMDs for vibrating footbridges under uncertainty conditions, the following numerical case-study is presented. Thus, the compliance of the VSLS of a numerical footbridge is guaranteed via the installation of a STMD. A detailed description of this numerical footbridge can be found in the French guidelines [2]. The structural behavior of the structure has been simulated via the finite element (FE) method. The FE package Ansys [15] has been used for this purpose. A numerical model using 646 
beam elements and 540 shell elements (BEAM188 and SHELL181 respectively) has been built (Fig. 3). A structural damping ratio of $\zeta_{f}=0.6 \%$ has been considered [2]. The numerical modal parameters of the footbridge have been obtained via a numerical modal analysis. As result of this analysis, it was checked that the first vertical vibration mode $\left(f_{f}=2.14 \mathrm{~Hz}\right)$ is prone to vibrate under pedestrian action (Fig. 3). The modal mass, $m_{f}$, of this vertical vibration mode is about $34706 \mathrm{~kg}$.

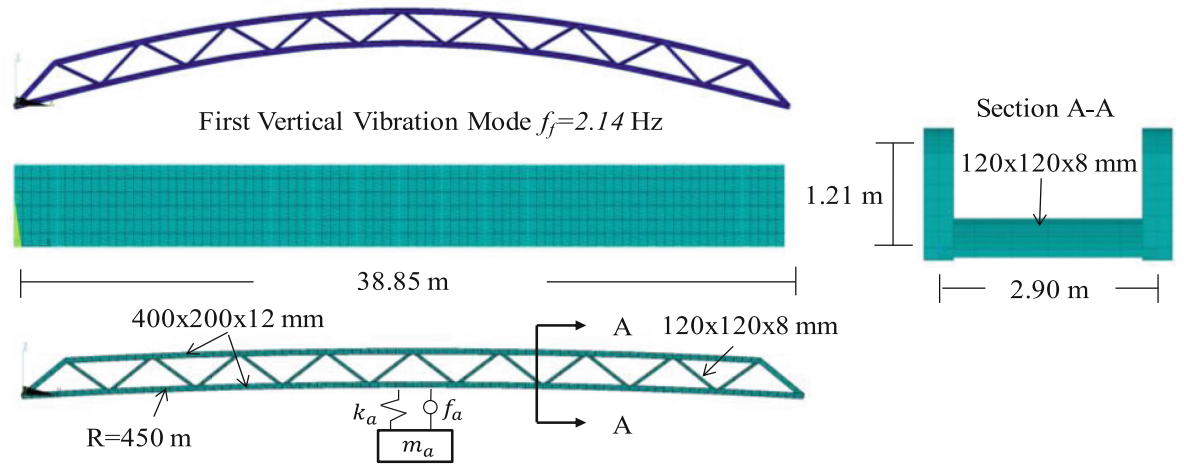

Fig. 3. Finite element model of the benchmark footbridge and first vertical vibration mode [2].

In order to check the VSLS of this footbridge, the recommendations of the French guidelines [2] have been considered herein. Thus, one design scenario has been taken into account with the following design parameters: (i) a pedestrian traffic of $1 \mathrm{P} / \mathrm{m}^{2}$ [2]; (ii) an allowable vertical acceleration, $\ddot{x}_{f, l i m}$, of $1.00 \mathrm{~m} / \mathrm{s}^{2}$ [2]; and (iii) an allowable reliability index, $\beta_{\text {lim }}$, of 1.5 [14]. If this requirement is not met, a STMD must be installed to reduce the amplitude of the pedestrian-induced vibrations according to the mentioned thresholds. The driving force, $f_{a}$, has been limited between the range $10-50 \mathrm{~N}$ $\left(f_{\min }-f_{\max }\right)$ in order to characterize adequately the behavior of the semi-active damper. According to the results provided by several researchers [3] a range of variation of $\pm 10 \%$ has been considered for both the first vertical natural frequency of the footbridge and its associated damping ratio. Consequently, it has been checked that the VSLS term of the multi-objective function is a random variable which follows a log-normal probability distribution. In order to obtain the reliability index, $\beta$, associated with the VSLS of the footbridge, a Monte Carlo simulation has been performed [10]. For the selection of the sample size (50000 simulations), a convergence analysis has been carried out. The mathematical package Matlab [12] has been employed for this study.

As result of this study, Table 1 shows the reliability index, $\beta$, associated with the VSLS without and with the STMD. As Table 1 shows, the motion-based design method allows control the dynamic response of the footbridge under uncertainty conditions without compromising the cost of the control system. Additionally, the parameters of the STMD designed according to this method have been included in Table 1. 
Table 1. Reliability index, $\beta$, of the VSLS of the footbridge (without and with STMD).

\begin{tabular}{l|l|l|l|l|l|l|l}
\hline STMD & $\ddot{x}_{f}\left[\mathrm{~m} / \mathrm{s}^{2}\right]$ & $\beta[-]$ & $\alpha_{d}[-]$ & $\beta_{d}[-]$ & $m_{a}[\mathrm{~kg}]$ & $k_{a}[\mathrm{~N} / \mathrm{m}]$ & $\left|f_{a}\right|[\mathrm{N}]$ \\
\hline No & - & -0.53 & - & - & - & - & - \\
\hline Yes & 1.00 & 1.50 & 224.19 & $771.6410^{-8}$ & 625 & $1.0810^{5}$ & 50 \\
\hline
\end{tabular}

\section{Conclusions}

In this manuscript, the motion-based design method has been presented and further implemented for the design of STMDs when they are employed to control the pedestrian-induced vibrations in footbridges under uncertainty conditions. According to this method, the design problem may be formulated via two coupled sub-problems: (i) a multi-objective optimization sub-problem; and (ii) a reliability analysis sub-problem. Thus, the multi-objective function of the problem is defined in terms of the parameters of the STMD which are needed to be determined and a reliability index which establishes the probability of compliance of the VSLS of the footbridge. Sampling techniques, as the Monte Carlos simulation method, are usually used to estimate numerically this reliability index. As application example, a footbridge, which is prone to vibrate due to walking pedestrian action, has been regarded. A STMD has been installed at its mid-span to reduce the pedestrian-induced vibrations. The STMD parameters have been obtained via the implementation of the mentioned proposal. As result of this study, the performance of the proposed method has been shown up. In spite of its good performance, further studies are needed, both to better determine the probabilistic distribution function of the different modal properties and to assess experimentally the performance of the structure designed according to this proposal.

Acknowledgements. This work was partially funded by two research projects: (i) research project RTI2018-094945-B-C21 (Ministerio de Economía y Competitividad of Spain and the European Regional Development Fund) and (ii) research project SEED-SD RTI2018-099639-B-I00 (Ministerio de Ciencia, Innovación y Universidades of Spain).

\section{References}

1. Van Nimmen, K., Lombaert, G., De Roeck, G., Van den Broeck, P.: Vibration serviceability of footbridges: evaluation of the current codes of practice. Eng. Struct. 59, 448-461 (2014)

2. Setra/AFGC Guide méthodologique passerelles piétonnes (Technical Guide Footbridges: Assessment of vibration behaviour of footbridge under pedestrian loading) (2006)

3. Hu, W.H., Caetano, E., Cunha, A.: Structural health monitoring of a stress-ribbon footbridge. Eng. Struct. 57, 578-593 (2013)

4. Weber, F., Feltrin, G., Huth, O.: Guidelines for Structural Control. Structural Engineering Research Laboratory. Swiss Federal Laboratories for Materials Testing Research. Dübendort, Switzerland (2006)

5. Moutinho, C.: Testing a simple control law to reduce broadband frequency harmonic vibrations using semi-active tuned mass dampers. Smart Mater. Struct. 24, 055007 (2015) 